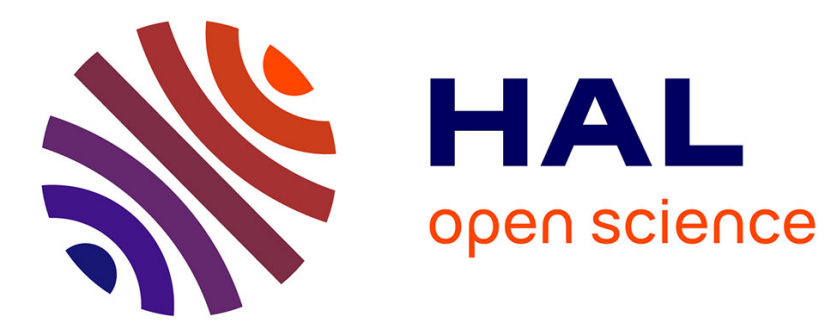

\title{
Synthesis and simulation of fractional orthonormal bases
}

\author{
Mohamed Aoun, Rachid R. Malti, Alain Oustaloup
}

\section{To cite this version:}

Mohamed Aoun, Rachid R. Malti, Alain Oustaloup. Synthesis and simulation of fractional orthonormal bases. 16th IFAC World Congress, Jul 2005, Prague, Czech Republic. pp.02747. hal-00401608

\section{HAL Id: hal-00401608 \\ https://hal.science/hal-00401608}

Submitted on 3 Jul 2009

HAL is a multi-disciplinary open access archive for the deposit and dissemination of scientific research documents, whether they are published or not. The documents may come from teaching and research institutions in France or abroad, or from public or private research centers.
L'archive ouverte pluridisciplinaire HAL, est destinée au dépôt et à la diffusion de documents scientifiques de niveau recherche, publiés ou non, émanant des établissements d'enseignement et de recherche français ou étrangers, des laboratoires publics ou privés. 


\title{
SYNTHESIS AND SIMULATION OF FRACTIONAL ORTHONORMAL BASES
}

\author{
Mohamed Aoun* Rachid Malti* Alain Oustaloup* \\ * LAP - UMR 5131 CNRS - Université Bordeaux I - \\ ENSEIRB, 351 cours de la Libération, 33405 Talence \\ cedex, France. \\ \{aoun, malti, oustaloup\}@lap.u-bordeaux1.fr
}

\begin{abstract}
This paper proposes a method for synthesis and simulation of fractional Laguerre, Kautz, and GOB functions. As compared to classical simulation methods, the new one take advantage of recursivity to improve the simulation schema.
\end{abstract}

Keywords: simulation, orthogonal functions, generalized orthogonal basis, fractional calculus, fractional derivative, dynamical system, identification.

\section{INTRODUCTION AND MATHEMATICAL BACKGROUND}

\subsection{Context and motivation}

Recently, we have proposed an interpolation of Laguerre functions to fractional differentiation orders which keeps them convergent when differentiation orders are non-integers (Aoun et al., 2003b). Then, we generalized the use of fractional orthogonal bases to any number of poles: real or by pair complex conjugate. It interpolates the well-known definition of the generalized orthogonal basis to fractional derivatives (Malti et al., 2004).

A major difficulty with fractional models, and therefore fractional bases, is their time-domain simulation. Often, the analytical solution of a model's output is not simple to compute. During the last 20 years numerical algorithms have been developed using either continuous or discrete-time rational models approximating fractional systems: (Oustaloup, 1983; Chen et al., 2003; Aoun et $a l ., 2003 a)$. In this paper, after recalling the orthogonalization procedure, we propose a new simulation diagram appropriate for evaluating of the output of fractional bases.

\subsection{Representation of fractional systems}

Fractional mathematical models are based on fractional differential equations:

$$
\begin{aligned}
& y(t)+b_{1} \mathbf{D}^{\beta_{1}} y(t)+\cdots+b_{m_{B}} \mathbf{D}^{\beta_{m_{B}}} y(t)= \\
& a_{0} \mathbf{D}^{\alpha_{0}} u(t)+a_{1} \mathbf{D}^{\alpha_{1}} u(t)+\cdots+a_{m_{A}} \mathbf{D}^{\alpha_{m_{A}}} u(t)
\end{aligned}
$$

where differentiation orders $\beta_{1}, \ldots, \beta_{m_{B}}, \alpha_{0}$, $\ldots, \alpha_{m_{A}}$ are allowed to be non-integer positive numbers. The concept of differentiation to an arbitrary order (non-integer),

$$
\mathbf{D}^{\gamma} \triangleq\left(\frac{d}{d t}\right)^{\gamma} \quad \forall \gamma \in R_{+}
$$

was defined in the $19^{t h}$ century. The main contribution to the establishment of the definition is due to Riemann and Liouville. They extend differentiation by using not only integer but also noninteger (real or complex) orders. The $\gamma$ fractional order derivative of $x(t)$ is defined as being an integer derivative of order $m=\lfloor\gamma\rfloor+1(\lfloor$.$\rfloor stands$ for the floor operator) of a non-integer integral of order $1-(m-\gamma)$ (Samko et al., 1993):

$$
\mathbf{D}^{\gamma} x(t) \triangleq \frac{1}{\Gamma(m-\gamma)}\left(\frac{d}{d t}\right)^{m} \int_{0}^{t} \frac{x(\tau) d \tau}{(t-\tau)^{1-(m-\gamma)}}
$$


where $t>0, \gamma>0$.

A more concise algebraic tool can be used to represent such fractional systems: the Laplace transform. The Laplace transform of a $\gamma$ order derivative $\left(\gamma \in \mathbb{R}_{+}\right)$of a signal $x(t)$ relaxed at $t=0$ (i.e. all derivatives of $x(t)$ equal 0 when $t<0$ ) is given by (Oldham and Spanier, 1974):

$$
\mathbf{L}\left\{\mathbf{D}^{\gamma} x(t)\right\}=s^{\gamma} X(s)
$$

This property allows to write the fractional differential equation (1), provided all signals $u(t)$ and $y(t)$ are relaxed at $t=0$, in a transfer function form:

$$
F(s)=\frac{\sum_{i=0}^{m_{A}} a_{i} s^{\alpha_{i}}}{1+\sum_{j=1}^{m_{B}} b_{j} s^{\beta_{j}}}
$$

where $\left(a_{i}, b_{j}\right) \in \mathbb{C}^{2}, \quad\left(\alpha_{i}, \beta_{j}\right) \in \mathbb{R}_{+}^{2}, \forall i=$ $0,1, \ldots, m_{A}, \forall j=1,2, \ldots, m_{B}$.

Definition 1. A transfer function $F(s)$ is commensurate of order $\gamma$ iff it can be written as $F(s)=$ $S\left(s^{\gamma}\right)$, where $S=\frac{T}{R}$ is a rational function with $T$ and $R$, two coprime polynomials.

All differentiation orders are multiples of the commensurate order, allowing to obtain a rational transfer function. In this paper, the commensurate order is left free to vary in $\mathbb{R}_{+}^{*}$. Taking as an example $F(s)$ defined in (3), assuming that $F(s)$ is commensurate of order $\gamma$, and using $F(s)=S\left(s^{\gamma}\right)$, one can write:

$$
S(s)=\frac{T(s)}{R(s)}=\frac{\sum_{m=0}^{m_{A}} a_{m} s^{\frac{\alpha_{m}}{\gamma}}}{1+\sum_{m=1}^{m_{B}} b_{m} s^{\frac{\beta_{m}}{\gamma}}}
$$

All powers of $s$ in (4) are integers. A sufficient condition for $F(s)$ to be commensurate is that all differentiation (or integration) orders belong to the set of rational numbers $\mathbb{Q}$. It covers a wide range of fractional systems.

\subsection{Stability condition}

Matignon (1998, theorem 2.21 p.150) has established the stability condition of any commensurate explicit fractional model. However, here is a revisited version of his theorem :

Theorem 2. A commensurate (of order $\gamma$ ) transfer function $F(s)=S\left(s^{\gamma}\right)=\frac{T\left(s^{\gamma}\right)}{R\left(s^{\gamma}\right)}$ is BIBO stable iff

$$
0<\gamma<2
$$

and for every $s \in \mathbb{C}$ such that $R(s)=0$

$$
|\arg (s)|>\gamma \frac{\pi}{2}
$$

\subsection{Fractional transfer functions belonging to $\mathrm{H}_{2}\left(\mathbb{C}^{+}\right)$}

Contrary to rational systems, stability condition does not guarantee for a fractional transfer function to belong to $H_{2}\left(\mathbb{C}^{+}\right)$. The $H_{2}$ norm of fractional systems was extensively studied in (Malti et al., 2003), where it was demonstrated that a fractional transfer function as defined in (3) belongs to $H_{2}\left(\mathbb{C}^{+}\right)$iff stability conditions $(5)$ and $(6)$ are satisfied and:

$$
\beta_{m_{B}}-\alpha_{m_{A}}>\frac{1}{2}
$$

Condition (7) will be necessary when choosing fractional generating functions for orthogonal bases to be synthesized.

\subsection{Scalar product, orthogonality and rational orthogonal functions}

The classical Laguerre, Kautz, and GOB functions form complete orthonormal basis in $L_{2}$ [0, $\infty$, according to the usual definition of the scalar product (G., 1975):

$$
\left\langle l_{n}(t), l_{m}(t)\right\rangle=\int_{0}^{\infty} l_{n}(t) l_{m}(t) d t=\delta_{n m}
$$

which reciprocal in the frequency domain is obtained by Plancherel's theorem:

$$
\left\langle L_{n}(s), L_{m}(s)\right\rangle=\frac{1}{2 \pi} \int_{-\infty}^{\infty} L_{n}(j \omega) \overline{L_{m}(j \omega)} d \omega=\delta_{n m}
$$

Any function $f(t) \in L_{2}[0, \infty[$, thus satisfying:

$$
\langle f(t), f(t)\rangle^{\frac{1}{2}}=\|f\|_{2}<\infty
$$

can be written as a linear combination of these functions:

$$
F(s)=\sum_{n=0}^{\infty} a_{n} L_{n}(s)
$$

$F(s)$ is the Laplace transform of $f(t)$. Usually, (11) is truncated to a given order $N$ which is justified by the fact that Fourier coefficients are convergent as $n$ tends to infinity. $F(s)$ is hence approximated by the finite sum:

$$
F(s) \approx F_{N}(s)=\sum_{n=0}^{N} \theta_{n} L_{n}(s)
$$

\section{ORTHONORMAL BASES CONSTRUCTIONS}

\subsection{Gram-Schmidt principle}

Given an arbitrary set of functions $\left\{F_{m}\right\}_{m=1}^{M}$, where $F_{m} \in H^{2}\left(\mathbb{C}^{+}\right) \forall m$, orthonormal functions 
$\left\{G_{m}\right\}_{m=1}^{M}$ are obtained, according to the GramSchmidt orthogonalisation principle, as a linear combination of generating functions $F_{m}, m=$ $1 \ldots M$ :

$$
\mathbf{G}=\Delta * \mathbf{F}
$$

where $\Delta$ is a real-valued $M \mathrm{x} M$ matrix,

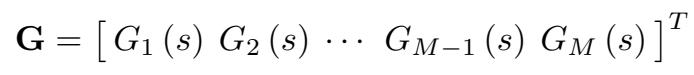

and

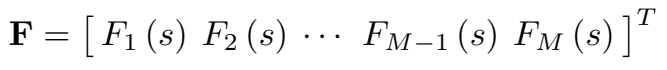

Since $\left\{G_{m}\right\}_{m=1}^{M}$ is the set of orthonormal functions

$$
\left\langle\mathbf{G}, \mathbf{G}^{T}\right\rangle=\mathbf{I}
$$

I denotes an $M$ by $M$ identity matrix. Thus, using (13):

$$
\left\langle\mathbf{G}, \mathbf{G}^{T}\right\rangle=\Delta\left\langle\mathbf{F}, \mathbf{F}^{T}\right\rangle \Delta^{T}=I
$$

Then, it is easy to check that

$$
\Delta^{T} \Delta=\left\langle\mathbf{F}, \mathbf{F}^{T}\right\rangle^{-1}
$$

From this quadratic form, $\Delta$, a lower triangular matrix, is computed using the Cholesky decomposition.

$$
\Delta=\text { Cholesky }\left(\left\langle\mathbf{F}, \mathbf{F}^{T}\right\rangle^{-1}\right)
$$

Using (13), functions of the orthonormal set are given by:

$$
\mathbf{G}=\text { Cholesky }\left(\left\langle\mathbf{F}, \mathbf{F}^{T}\right\rangle^{-1}\right) \times \mathbf{F}
$$

The remaining difficulty is to compute the matrix of scalar products $\left\langle\mathbf{F}, \mathbf{F}^{T}\right\rangle$ for fractional transfer functions which are known to be multivalued complex functions as soon as non-integer differentiation is involved. Hence, a plane cut is necessary in the complex $s$-plane. A procedure for computing the scalar product of any fractional explicit transfer function is described in appendix A.

\subsection{Fractional Generating functions}

The construction of the orthogonal basis starts by choosing a set of generating functions which must not be colinear in the sense of the definition (9) of the scalar product. Each generating function can introduce either a real mode or a complex one. If a generating function introduces a complex mode, then the next generating function must introduce its conjugate so that the impulse response remains a real signal. Once these functions chosen, (17) is applied so that the orthogonal functions $G_{m}$ 's are obtained.
2.2.1. Fractional Laguerre-like generating functions If a real mode is to be included in the first FraGOB, a generating function is chosen as:

$$
F_{m_{0}}(s)=\frac{1}{\left(s^{\gamma}+\lambda_{m_{0}}\right)^{m_{0}}}
$$

where

$$
m_{0}=\left\lfloor\frac{1}{2 \gamma}\right\rfloor+1
$$

and

$$
\left.\lambda_{m_{0}} \in \mathbb{R}_{+}^{*}, \quad \gamma \in\right] 0,2[
$$

All other generating functions to be included with real valued modes are chosen as:

$$
F_{m}(s)=F_{m-1}(s) \frac{1}{\left(s^{\gamma}+\lambda_{m}\right)}
$$

where

$$
\left.\lambda_{m} \in \mathbb{R}_{+}^{*}, \quad \forall m \in \mathbb{N}, \quad m \geq m_{0}, \quad \gamma \in\right] 0,2[
$$

Conditions announced in (20 and 22) stem from stability theorem 2 and the fact that impulse responses of (18 and 21) are real-valued signals.

Condition (19) stems from the fact that each generating function must belong to $H^{2}\left(\mathbb{C}^{+}\right)$. Hence, applying (7) for the generating function (18) shows that:

$$
\gamma m_{0}>\frac{1}{2}
$$

Keeping in mind that $m_{0}$ is integer yields (19).

It is interesting to point out that, in the special case where all the modes $\lambda_{m}$ are chosen to be alike, the multiplicity of the mode $\lambda_{m}$ is incremented in (21) for every new function, in which case the set of fractional Laguerre generating functions is obtained as illustrated in (Aoun et al., 2003b).

\subsubsection{Fractional Kautz-like generating functions} Suppose that $(n+1)$ modes $\lambda_{m_{0}}, \ldots, \lambda_{m_{0}+n}$ have been included in $F_{m_{0}}, F_{m_{0}+1}, \ldots, F_{m_{0}+n}$ and now we wish to include a complex mode $\lambda_{m_{0}+n+1}$. Then, a conjugate mode must follow $\left(\lambda_{m_{0}+n+2}=\right.$ $\left.\overline{\lambda_{m_{0}+n+1}}\right)$ in order to have a real impulse response. Moreover, both basis functions $F_{m_{0}+n+1}$ and $F_{m_{0}+n+2}$ are replaced by two new basis functions $F_{m_{0}+n+1}^{\prime}$ and $F_{m_{0}+n+1}^{\prime \prime}$ which have real impulse responses and which are a linear combination of $\frac{1}{\left(s^{\gamma}+\lambda_{r}\right)^{r}}$ and $\frac{1}{\left(s^{\gamma}+\overline{\lambda_{r}}\right)^{r}}$, where $r=m_{0}+n+1$.

The linear combination we are suggesting can be expressed as:

$$
\left[\begin{array}{c}
F_{r}^{\prime} \\
F_{r}^{\prime \prime}
\end{array}\right]=\left[\begin{array}{ll}
c_{0} & c_{1} \\
c_{0}^{\prime} & c_{1}^{\prime}
\end{array}\right]\left[\begin{array}{l}
\frac{1}{\left(s^{\gamma}+\lambda_{r}\right)^{r}} \\
\frac{1}{\left(s^{\gamma}+\overline{\lambda_{r}}\right)^{r}}
\end{array}\right] F_{r-1}
$$

where $c_{0}, c_{1}, c_{0}^{\prime}, c_{1}^{\prime} \in \mathbb{C}$.

As long as Gram-Shmidt orthogonalization procedure will follow, the only constraint while choosing $c_{0}, c_{1}, c_{0}^{\prime}$ and $c_{1}^{\prime}$ is that both functions $F_{r}^{\prime}$ and $F_{r}^{\prime \prime}$ 
have real-valued impulse responses which gives the following conditions:

$$
c_{0}=\overline{c_{1}} \quad \text { and } \quad c_{0}^{\prime}=\overline{c_{1}^{\prime}}
$$

Four more degrees of freedom are left while choosing the real and imaginary parts of $c_{0}, c_{1}, c_{0}^{\prime}$ and $c_{1}^{\prime}$. Therefore, as a result any of the following transfer functions can be chosen as generating functions of the basis:

$$
\begin{aligned}
F_{r}^{\prime}(s) & =\frac{\left(\beta s^{\gamma}+\mu\right)}{s^{2 \gamma}+\left(\lambda_{r}+\overline{\lambda_{r}}\right) s^{\gamma}+\lambda_{r} \overline{\lambda_{r}}} F_{r-1} \\
F_{r}^{\prime \prime}(s) & =\frac{\left(\beta^{\prime} s^{\gamma}+\mu^{\prime}\right)}{s^{2 \gamma}+\left(\lambda_{r}+\overline{\lambda_{r}}\right) s^{\gamma}+\lambda_{r} \overline{\lambda_{r}}} F_{r-1}
\end{aligned}
$$

where

$$
\left.\left|\arg \left(\lambda_{r}\right)\right|>\gamma \frac{\pi}{2}, \quad \gamma \in\right] 0,2[,
$$

and $(\beta, \mu) \neq d\left(\beta^{\prime}, \mu^{\prime}\right), \forall d \in \mathbb{C}$ and $\left(\beta, \mu, \beta^{\prime}, \mu^{\prime}\right) \in$ $\mathbb{R}^{4}$. Parameters $\left(\beta, \mu, \beta^{\prime}, \mu^{\prime}\right)$ can be chosen arbitrarily with the following constraint $(\beta, \mu) \neq$ $d\left(\beta^{\prime}, \mu^{\prime}\right), \forall d \in \mathbb{C}$ i.e. $F_{r}^{\prime}(s)$ and $F_{r}^{\prime \prime}(s)$ must not be co-linear according to the definition (9) of the scalar product. One should, however, keep in mind that an infinite pair of functions can span a plane. Hence, two different choices of $\left(\beta, \mu, \beta^{\prime}, \mu^{\prime}\right)$ may lead to different pairs of orthogonal functions $F_{r}^{\prime}(s)$ and $F_{r}^{\prime \prime}(s)$.

When $r=1$, the two first functions are :

$$
\begin{aligned}
F_{1}^{\prime}(s) & =\left(\frac{\left(\beta s^{\gamma}+\mu\right)}{s^{2 \gamma}+\left(\lambda_{1}+\overline{\lambda_{1}}\right) s^{\gamma}+\lambda_{1} \overline{\lambda_{1}}}\right)^{m_{0}} \\
F_{2}^{\prime \prime}(s) & =\left(\frac{\left(\beta^{\prime} s^{\gamma}+\mu^{\prime}\right)}{s^{2 \gamma}+\left(\lambda_{1}+\overline{\lambda_{1}}\right) s^{\gamma}+\lambda_{1} \overline{\lambda_{1}}}\right)^{m_{0}}
\end{aligned}
$$

where $m_{0}$ is such as $F_{1}^{\prime}$ and $F_{2}^{\prime \prime}$ belong to $H^{2}\left(\mathbb{C}^{+}\right)$. It is easy to show that $m_{0}$ is given by (19).

In the special case where all complex conjugate modes $\left(\lambda_{r}, \bar{\lambda}_{r}\right)$ are chosen to be alike, the set of fractional Kautz-like basis is synthesized.

Remark Completeness of the FraGOB is yet to be proven. However, the completeness of fractional Laguerre basis is proved in (Malti et al., 2004). Then, when conditions (22) is satisfied and all the poles are chosen to be alike:

$$
\lambda_{m_{0}}=\lambda_{m_{0}+1}=\lambda_{m_{0}+2}=\ldots=\lambda_{\infty},
$$

and $m_{0}=\left\lfloor\frac{1}{2 \gamma}\right\rfloor+1$, the fractional Laguerre basis is dense in $H^{2}\left(\mathbb{C}^{+}\right)$. Therefore, it can be used to model any finite energy fractional system.

\section{NUMERICAL SIMULATION OF FRACTIONAL GENERALIZED ORTHONORMAL BASES}

Since the filters of the fractional bases are irrational transfer functions, some simulation methods will be introduced for this class of transfer function.

\subsection{Simulation of irrational transfer functions}

Simulation of fractional systems is complicated due to their long memory behavior as shown by Oustaloup (1995). Many methods have been developed (Lin, 2001; Aoun et al., 2003a; Chen et al., 2003). Mainely, they are based on the approximation of a fractional model on either a rational discrete-time or a rational continuous-time model. In this paper, we detail some methods based on discrete-time models. The reader can find however a more detailed presentation in (Aoun et al., 2003a).

In these methods, the fractional differentiator $s^{\gamma}$ is substituted by its discrete-time equivalent.

$$
s^{\gamma} \rightarrow \psi\left(z^{-1}\right)
$$

As a result, a discrete-time transfer function is obtained:

$$
\begin{aligned}
\frac{Y(s)}{U(s)} & =\frac{b_{0}+b_{1} s^{\gamma}+b_{2} s^{2 \gamma}+\ldots+b_{n_{B}} s^{n_{B} \gamma}}{a_{0}+a_{1} s^{\gamma}+a_{2} s^{2 \gamma}+\ldots+a_{n_{A}} s^{n_{A} \gamma}}, \\
& \equiv \frac{Y\left(z^{-1}\right)}{U\left(z^{-1}\right)}, \\
& \equiv \frac{b_{0}+b_{1} \psi\left(z^{-1}\right)+\ldots+b_{n_{B}}\left(\psi\left(z^{-1}\right)\right)^{n_{B}}}{a_{0}+a_{1} \psi\left(z^{-1}\right)+\ldots+a_{n_{A}}\left(\psi\left(z^{-1}\right)\right)^{n_{A}}} .
\end{aligned}
$$

$\psi\left(z^{-1}\right)$, the discrete mapping of the Laplace operator $s$, can be computed using various approximation methods. The most common are Euler's, Tustin's, Simpson's, or Al-Alaoui's (Al-Alaoui, 1994; Vinagre et al., 2000; Oustaloup, 1995). These analogue-to-digital open-loop design methods lead to irrational z-transforms which are then approximated either by a truncated Taylor series expansion or a continuous fraction expansion. The obtained digital model can then be simulated using a classical implementation structure: directform, parallel-form, cascade-form, lattice-form, ... Euler:

$$
\begin{gathered}
\psi\left(z^{-1}\right)=\left(\frac{1-z^{-1}}{T s}\right)^{\gamma} \\
=\left(\frac{1}{T s}\right)^{\gamma}\left(1-\gamma z^{-1}+\frac{\gamma(\gamma-1)}{2} z^{-2}+\ldots\right) \\
=\left(\frac{1}{T s}\right)^{\gamma} \sum_{k=0}^{\infty}\left((-1)^{k}\left(\begin{array}{l}
\gamma \\
k
\end{array}\right) z^{-k}\right)
\end{gathered}
$$

Tustin:

$$
\begin{aligned}
& \psi\left(z^{-1}\right)=\left(\frac{2}{T s}\right)^{\gamma}\left(\frac{1-z^{-1}}{1+z^{-1}}\right)^{\gamma} \\
& =\left(\frac{2}{T s}\right)^{\gamma}\left(1-2 \gamma z^{-1}+2 \gamma^{2}(\gamma-1) z^{-2}+\ldots\right)
\end{aligned}
$$


Simpson:

$$
\begin{aligned}
& \psi\left(z^{-1}\right)=\left(\frac{3}{T s}\right)^{\gamma}\left(\frac{\left(1-z^{-1}\right)\left(1+z^{-1}\right)}{1+4 z^{-1}+z^{-2}}\right)^{\gamma} \\
& =\left(\frac{3}{T s}\right)^{\gamma}\left(1-4 \gamma z^{-1}+2 \gamma(4 \gamma+3) z^{-2}+\ldots\right)
\end{aligned}
$$

\section{Al-Alaoui:}

$$
\begin{aligned}
& \psi\left(z^{-1}\right)=\left(\frac{8}{7 T s}\right)^{\gamma}\left(\frac{1-z^{-1}}{1+\frac{z^{-1}}{7}}\right)^{\gamma} \\
= & \left(\frac{8}{7 T s}\right)^{\gamma}\left(1-\frac{8 \gamma}{7} z^{-1}+\frac{-24 \gamma+32 \gamma^{2}}{49} z^{-2}+\ldots\right)
\end{aligned}
$$

\subsection{Simulation Diagram of FraGOB}

Let $F$ be a dynamic system approximated using a FraGOB :

$$
F(s)=\sum_{m=m_{0}}^{M} \theta_{m} G_{m}(s)
$$

The time response of $F$ can be evaluated by simulating directly the filters $G_{m}$. The main drawback of this method is that each $G_{m}$ is simulated separately. Given the complexity of the $G_{m}$ expression, specially when $m$ increases, simulation is slow and hard to achieve.

To avoid such problems, since orthonormal filters $G_{m}$ are linear combinations of generating functions $F_{m}$, and since each generating function $F_{m}$ is a product of $F_{m-1}$ by a fractional mode, one can use the simulation diagram (1). The coefficients $\delta_{i, j}$ are elements of the matrix which stems from the Cholesky decomposition (16).

\section{EXAMPLE}

Let $\gamma=0.8$ be the fractional order and all five first eigenvalues of the basis $\left(2,2 e^{ \pm j \frac{\pi}{3}}, 1\right.$ and 0.5$)$.

Since $\gamma=0.8$, the index of the first generating base is $m_{0}=\left\lfloor\frac{1}{2 \times 0.8}\right\rfloor+1=1$. Then, by applying (18), $F_{1}$ is given by :

$$
F_{1}(s)=\frac{1}{s^{0.8}+2}
$$

The second and the third functions include two complexe conjugate modes. Then, $F_{3}$ and $F_{4}$ are obtained with (28) and (29). In this example, we fixed arbitrarily $\beta=\mu^{\prime}=0$ and $\beta^{\prime}=\mu=1$ so that the two functions are not co-linear.

$$
\begin{aligned}
& F_{2}(s)=\frac{F_{1}(s)}{s^{1.6}+0.5 s^{0.8}+4} \\
& F_{3}(s)=\frac{s^{0.8} F_{1}(s)}{s^{1.6}+0.5 s^{0.8}+4}
\end{aligned}
$$

The forth and the fifth generating functions introduce respectively the two reals modes 1 and 0.5 . $F_{4}$ and $F_{5}$ are then given by $(21)$.

$$
F_{4}(s)=\frac{F_{3}(s)}{s^{0.8}+1} \quad \text { and } \quad F_{5}(s)=\frac{s^{0.8} F_{4}(s)}{s^{0.8}+0.5}
$$

The scalar product matrix $\left\langle\mathbf{F}, \mathbf{F}^{\mathbf{T}}\right\rangle$ is computed using algorithm developed in the appendix (A) :

$$
\left\langle\mathbf{F}, \mathbf{F}^{\mathbf{T}}\right\rangle=\left[\begin{array}{ccccc}
341.53 & 19.58 & 43.57 & 12.40 & 5.20 \\
19.58 & 6.32 & 2.11 & 2.78 & 2.12 \\
43.58 & 2.11 & 12.27 & 3.28 & 0.16 \\
12.40 & 2.78 & 3.28 & 2.19 & 0.78 \\
5.20 & 2.12 & 0.16 & 0.78 & 1.01
\end{array}\right] 10^{-3}
$$

The matrix $\boldsymbol{\Delta}$ stem from the Cholesky decomposition is then obtained :

$$
\boldsymbol{\Delta}=\left[\begin{array}{ccccc}
1.71 & 0 & 0 & 0 & 0 \\
0.79 & -13.87 & 0 & 0 & 0 \\
-1.61 & 0.91 & 12.23 & 0 & 0 \\
-1.16 & 21.10 & 13.94 & -50.29 & 0 \\
-0.03 & -23.14 & 1.96 & 5.06 & 60.65
\end{array}\right]
$$

The vectors of the orthonormal basis are linear combinations of $F_{m}$ and they are computed by applying formula (17).

$$
\begin{gathered}
G_{1}(s)=\frac{1.71}{s^{0.8}+2} \\
G_{2}(s)=\frac{0.79 s^{1.6}+1.59 s^{0.8}-10.69}{\left(s^{0.8}+2\right)\left(s^{1.6}+0.5 s^{0.8}+4\right)} \\
G_{3}(s)=\frac{-1.61 s^{1.6}+9.01 s^{0.8}-5.54}{\left(s^{0.8}+2\right)\left(s^{1.6}+0.5 s^{0.8}+4\right)} \\
G_{4}(s)=\frac{-1.16 s^{2.4}+10.45 s^{1.6}-22.23 s^{0.8}+16.45}{\left(s^{0.8}+1\right)\left(s^{0.8}+2\right)\left(s^{1.6}+0.5 s^{0.8}+4\right)} \\
G_{5}(s)= \\
\frac{-0.03 s^{3.2}+1.85 s^{2.4}-15.37 s^{1.6}+29.24 s^{0.8}-11.63}{\left(s^{0.8}+0.5\right)\left(s^{0.8}+1\right)\left(s^{0.8}+2\right)\left(s^{1.6}+0.5 s^{0.8}+4\right)}
\end{gathered}
$$

The diagram (figure (1)) is used to simulate the step response of the five orthonogonal functions between 0 and $T_{f}=30 s$ with the sampling period $T_{s}=0.1 s$. The Laplace variable is substituted with the series expansion of the Euler approximation (35). As the input signal is null for negative time, the series in (35) is truncated to the number of input samples $\frac{T_{f}}{T_{s}}=300$. The step responses of the orthogonal functions are plotted on figure 2 .

\section{CONCLUSION}

A new simulation diagram of fractional systems approximated on continuous-time FraGOB (Fractional Generalized Orthogonal basis) is presented. Numerical simulation of the elementary transfer functions of the diagram is carried using classical approximations like Euler, Tusin, Simpson. 


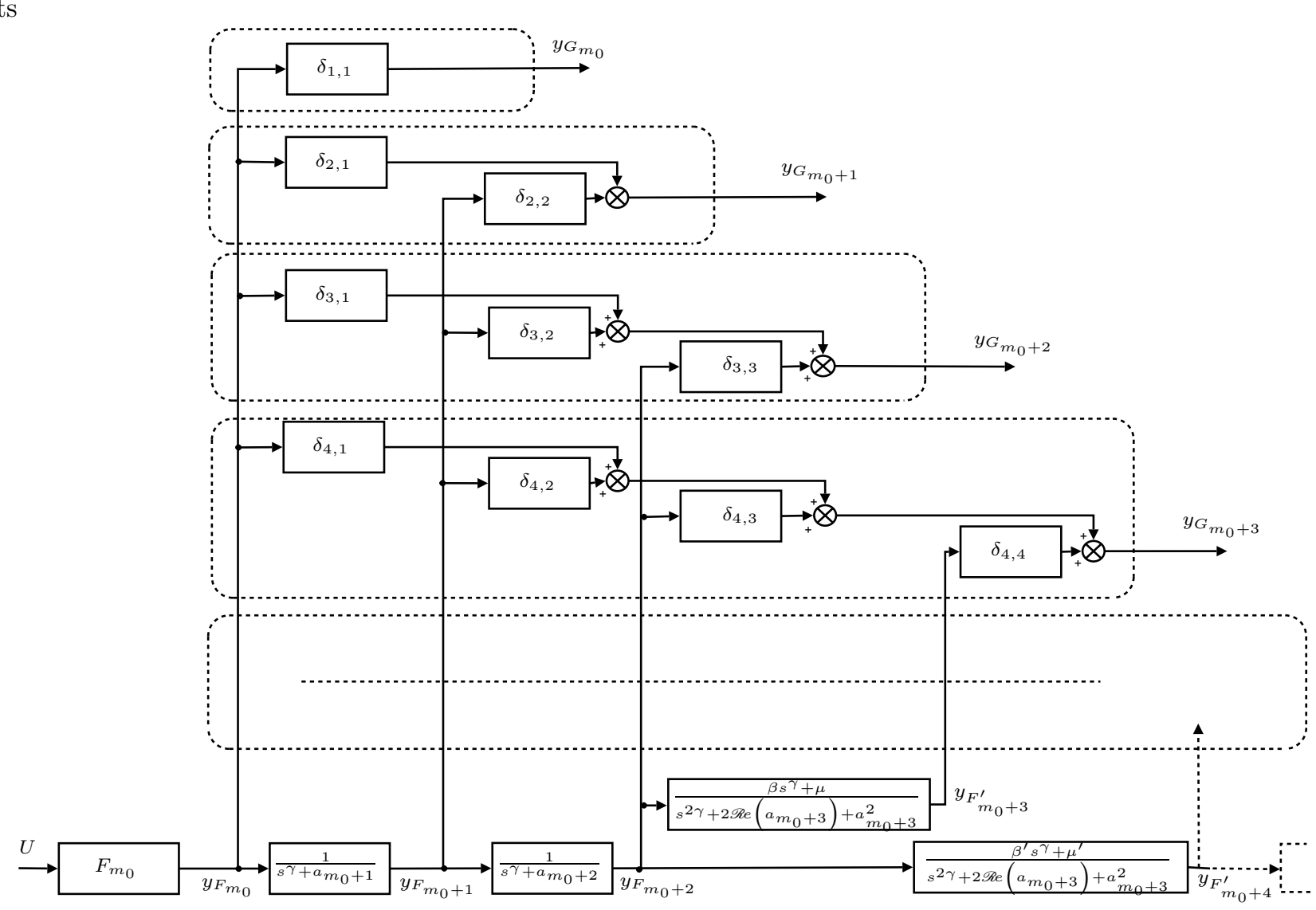

Fig. 1. simulation diagram of fraGOB

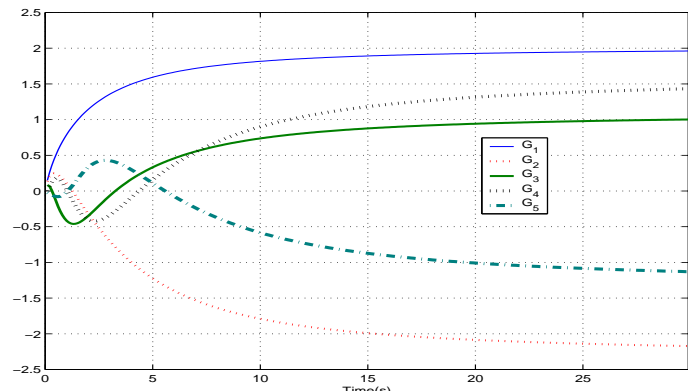

Fig. 2. Step responses of $G_{1}, G_{2}, G_{3}, G_{4}$ and $G_{5}$

\section{Appendix A. SCALAR PRODUCT OF ANY PAIR OF FRACTIONAL EXPLICIT TRANSFER FUNCTIONS}

Assume any pair $(G(s), H(s))$ of fractional explicit stable transfer functions. Then, the scalar product between these two functions is expressed in the frequency domain as:

$$
\langle G, H\rangle=\frac{1}{2 \pi} \int_{-\infty}^{\infty} G(j \omega) \overline{H(j \omega)} d \omega
$$

Define the following change of variable, where $\gamma$ is the commensurate order:

$$
x=\omega^{\gamma} \Rightarrow d \omega=\frac{1}{n} x^{\frac{1}{\gamma}-1} d x
$$

Define $q$ and $\rho$ respectively as integer and noninteger parts of $\frac{1}{\gamma}$. Then,

$$
\langle G, H\rangle=\frac{1}{\pi n} \int_{0}^{\infty} x^{q+\rho-1} \frac{A(x)}{B(x)} d x
$$

Two cases are distinguished:

$A .1 \operatorname{deg}(B) \leq \operatorname{deg}(A)+\frac{1}{n}$

In this case,

$$
\langle G, H\rangle=\infty
$$

because the order of numerator in (A.3) is greater than the order of denominator. This case is encountered for stable fractional systems when condition (7) is not satisfied.

$A .2 \operatorname{deg}(B)>\operatorname{deg}(A)+\frac{1}{n}$

Depending on the nullity of $\rho$, the solution to integral (A.1) is different. Again, two cases are distinguished.

A.2.1. $0<\rho<1$ This condition means that $\frac{1}{\gamma}$ is non-integer. A partial fraction expansion is carried out on $x^{q} \frac{A(x)}{B(x)}$ and gives: 


$$
x^{\rho-1}\left[x^{q} \frac{A(x)}{B(x)}\right]=\sum_{k=1}^{r} \sum_{l=1}^{v_{k}} \frac{a_{k, l} x^{\rho-1}}{\left(x+s_{k}\right)^{l}}
$$

Replacing back in (A.3), gives:

$$
\langle G, H\rangle=\frac{1}{n \pi} \sum_{k=1}^{r} \sum_{l=1}^{v_{k}} a_{k, l} s_{k}^{-l} \int_{0}^{\infty} \frac{x^{\rho-1} d x}{\left(1+s_{k}^{-1} x\right)^{l}}
$$

Similar function was integrated in (Erdélyi, 1954) and is also reported in (Gradshteyn and Ryshik, 1980, formula 3.194,4 p. 285 ). Plugging the result gives:

$$
\langle G, H\rangle=\frac{\sum_{k=1}^{r} \sum_{l=1}^{v_{k}}(-1)^{l-1} a_{k, l} s_{k}^{\rho-l}\left(\begin{array}{c}
\rho-1 \\
l-1
\end{array}\right)}{n \sin (\rho \pi)}
$$

A.2.2. $\rho=0$ This condition means that $\frac{1}{\gamma}$ is integer. The following expansion is carried out:

$$
\begin{gathered}
x^{q-1} \frac{A(x)}{B(x)}=\sum_{k=2}^{r} \frac{c_{k}}{\left(x+s_{1}\right)\left(x+s_{k}\right)}+ \\
\sum_{k=1}^{r} \sum_{l=2}^{v_{k}} \frac{b_{k, l}}{\left(x+s_{k}\right)^{l}}
\end{gathered}
$$

Where $s_{1}$ is an arbitrary chosen pole. After some tedious calculations, the following result is obtained:

$$
\begin{gathered}
\langle G, H\rangle=\sum_{k=2}^{r} \frac{c_{k}\left(\ln \left(s_{k}\right)-\ln \left(s_{1}\right)\right)}{n \pi\left(s_{k}-s_{1}\right)}+ \\
\sum_{k=1}^{r} \sum_{l=2}^{v_{k}} \frac{b_{k, l} s_{k}^{1-l}}{n \pi(l-1)}
\end{gathered}
$$

which completes the computations of scalar product of any fractional explicit transfer functions.

\section{REFERENCES}

Al-Alaoui, M.A (1994). Novel IIR differentiator from the simpson integration rule. IEEE Transactions on Circuits and Systems I. Fundamental Theory andApplications $\mathbf{4 1}$ (2), 186-187.

Aoun, M., Malti R., Levron F. and Oustaloup A. $(2003 a)$. Numerical simulations of fractional systems. In: In proceedings of ASME. Chicago, USA.

Aoun, M., R. Malti, F. Levron and A. Oustaloup $(2003 b)$. Orthonormal basis functions for modeling continuous-time fractional systems. In: SySId (Elsevier, Ed.). IFAC. Elsevier. Rotterdam, Netherlands.

Chen, Y.Q., Vinagre B. and Podlubny I. (2003). A new discretization method for fractional order differentiators via continued fraction expansion. In: ASME. Chicago, Illinois.

Erdélyi, A. et al. (1954). Tables of integral transforms. Vol. I and II. New-York.
G., Szego (1975). Orthogonal Polynomials. 4th edition ed.. American Mathematical Society.

Gradshteyn, I.S. and I.M. Ryshik (1980). Table of integrals, series, and products.

Lin, J. (2001). Modélisation et identification de systèmes d'ordre non entier. PhD thesis. Université de Poitiers.

Malti, R., M. Aoun, F. Levron and A. Oustaloup (2003). $H_{2}$ norm of fractional differential systems. In: Proceedings of ASME. number DETC2003/VIB-48387.

Malti, R., M. Aoun, F. Levron and A. Oustaloup (2004). Unified construction of fractional generalized orthogonal basis. In: First IFAC Workshop on Fractional Differentiation and its Applications, FDA, Bordeaux France.

Matignon, D. (1998). Stability properties for generalized fractional differential systems. ESAIM proceedings - Systèmes Différentiels Fractionnaires - Modèles, Méthodes et Applications 5, 145-158.

Oldham, K.B. and J. Spanier (1974). The fractionnal calculus. Academic Press, New-York and London.

Oustaloup, A. (1983). Systèmes asservis linéaires d'ordre fractionnaire. Masson - Paris.

Oustaloup, A. (1995). La dérivation non-entière: théorie, synthèse et applications. Hermès. Paris.

Samko, S.G., A.A. Kilbas and O.I. Marichev (1993). Fractional integrals and derivatives: theory and applications. Gordon and Breach Science.

Vinagre, B. M., I. Podlubny, A. Hernandez and V. Feliu (2000). Some approximations of fractional order operators used in control theory and applications. Fractional Calculus and Applied Analysis 3(3), 231-248. 